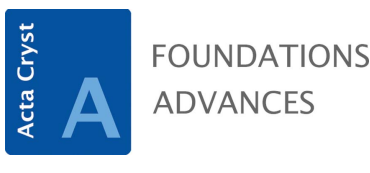

ISSN 2053-2733
Keywords: book review; group theory; periodic table.

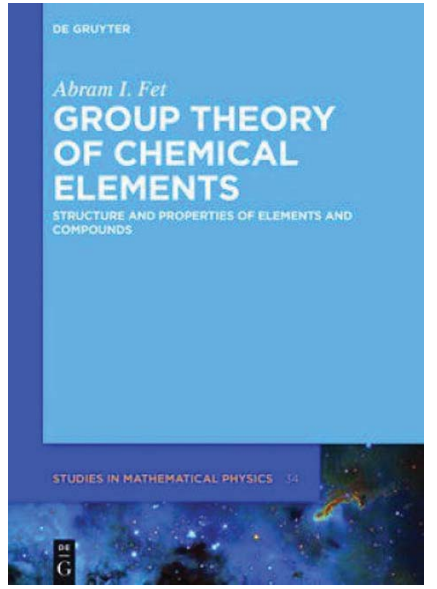

(C) 2018 International Union of Crystallography

\section{Group Theory of Chemical Elements. Structure and Properties of Elements and Compounds. By Abram I. Fet. De Gruyter, 2016. Pp. viii + 185. Price EUR 119.95/ USD 168.00/GBP 108.99 (hardcover). ISBN 978-3-11- 047518-0.}

\author{
Maurice Kibler* \\ Institut de Physique Nucléaire de Lyon, 43 boulevard du 11 novembre 1918, 69622 Villeurbanne Cedex, France. \\ *Correspondence e-mail: m.kibler@ipnl.in2p3.fr
}

This book is a translation (by Vladimir Slepkov) of a book written in Russian by Abram I. Fet. The Russian book goes back to the beginning of the 1980s, as is clear from the introduction to the book in Russian dated 9 January 1984.

The aim of the book is to give a classification of chemical elements and to describe some of their properties by using group-theoretical methods developed in the 1960s for the classification of elementary particles, more precisely strongly interacting particles (the so-called hadrons). The central part of the book (in line with the title of the book) consists of Chapters 5 and 6, in which group-theoretical methods are used for generating a new version of the Mendeleev periodic table (see Table 6.1) of chemical elements. The aim of the other chapters and appendices is to prepare the reader for the use of Lie groups and Lie algebras for the classification of neutral atoms. In particular, Chapter 4 presents the bases of the SU(3) eightfold way model, as developed for the classification of hadrons (baryons and mesons).

Contents of the book. Chapter 1 deals with group-theoretical methods used in quantum physics and quantum chemistry. The accent is put on the rotation groups $\mathrm{O}(3), \mathrm{SO}(3)$ and $\mathrm{SO}(4)$, and on the conformal group $\mathrm{SO}(4,2)$. This leads to the chain of groups $\mathrm{SO}(4,2) \supset$ $\mathrm{SO}(4) \supset \mathrm{SO}(3)$ of interest for the quantum system of a single particle in a central potential, like the electron in the hydrogen atom.

In Chapter 2, it is shown how a quantum system can be described by representations of a symmetry group. The connection between the representations of a group and a quantum system is based on the relation between the Hamiltonian of the system and the invariant operators (also called Casimir operators) of its symmetry group. It is thus possible to derive observables of a quantum system from its symmetry group. The cases of the Lorentz group $\mathrm{SO}(3,1)$ and of the Poincare group are briefly addressed.

Chapter 3 is concerned with some basic ingredients (topological structure, reducible and irreducible representations, tensor product of representations, universal enveloping algebra, Casimir operators) of Lie groups and Lie algebras in view of their application in Chapters 4, 5 and 6. In particular, some generalities are given around the groups $\mathrm{GL}(n, \mathbb{C}), \mathrm{U}(n), \mathrm{SU}(n), \mathrm{O}(n), \mathrm{SO}(n), \mathrm{SO}(p, q)$, the Euclidean isometry group $\mathrm{M}(3)$ and the Poincaré group.

Chapter 4 is devoted to the classification of particular elementary particles, i.e. hadrons [more specifically the octet of baryons $\left(\frac{1}{2}\right)^{+}$and the decuplet of baryons $\left(\frac{3}{2}\right)^{+}$], via the use of the group $\mathrm{SU}(3)$ and the chain of groups $\mathrm{SU}(6) \supset \mathrm{SU}(2) \times \mathrm{SU}(3)$. The notions of isospin, hypercharge and mass formula for baryons are introduced in connection with Cartan generators and Casimir operators of $\mathrm{SU}(6) \supset \mathrm{SU}(2) \times \mathrm{SU}(3)$. The chapter closes with general classification principles in quantum theory with a special emphasis on the classification of hadrons.

In Chapter 5, a group-theoretical description of chemical elements is set up by combining the empirical Madelung numbering of neutral atoms and a specific infinitedimensional unitary representation of the group $\mathrm{SO}(4,2)$. In this approach, $\mathrm{SO}(4,2)$ describes the system of atoms as a whole: all the chemical elements are considered as the states of a quantum system, the states being connected through actions of the group 
$\mathrm{SU}(2) \times \mathrm{SO}(4,2)$. For the purpose of comparison between theory and experiment, a mass formula is derived for the atomic weights.

Finally, the classification and some chemical properties of elements are examined in Chapter 6 on the basis of a chain of groups involving the direct product $\mathrm{SU}(2) \times \mathrm{SO}(4,2)$. Multiplets of elements are described by the various groups of the chain and operators of chemical affinity are derived. Contact between theory and experimental data is established for ionization potentials, atomic volumes, enthalpies of formation, heats of evaporation, energies of ionic lattices, bonding energies of diatomic molecules and dissociation energies of lanthanide compounds.

Appendix A concerns the $\mathrm{SO}(4)$ approach to the discrete energy spectrum of the hydrogen atom. This approach, developed by V. Fock in 1935, rests on the passage from the position representation ( $r$-representation) to the momentum representation ( $p$-representation) and on the stereographic projection of the sphere $S^{3}$ on the space $\mathbb{R}^{3}$.

Appendix B is centered on some linear representations of the groups $\mathrm{SU}(3)$ and $\mathrm{SO}(4)$ and on their restriction to their subgroups $\mathrm{SU}(2)$ and $\mathrm{SO}(3)$, respectively. The reductions $\mathrm{SU}(3) \rightarrow \mathrm{SU}(2)$ and $\mathrm{SO}(4) \rightarrow \mathrm{SO}(3)$ play a key role in the classification of hadrons and in the understanding of the discrete energy levels of the hydrogen atom, respectively.

Concluding remarks. The idea of transferring methods developed for the classification of hadrons (by M. Gell-Mann, Y. Neeman and S. Okubo) and nuclei (by E. P. Wigner, F. Gürsey, L. A. Radicati and A. Pais) to the classification of chemical elements is due to Yu. B. Rumer in 1970. This idea led Rumer and Fet in 1971 to show the relevance of the group $\mathrm{SU}(2) \times \mathrm{SO}(4)$ for a group-theoretical approach to the Mendeleev table. The SO(4) symmetry was extended by B. Konopel'chenko in 1972 to the group $\mathrm{SO}(4,2)$. This was the starting point for a series of seminal works by Rumer, Fet and collaborators on a qualitative and quantitative approach via the group $\mathrm{SU}(2) \times \mathrm{SO}(4,2)$ to the table of Mendeleev.

It is to be noted that the idea of using the group $\operatorname{SO}(4,2)$ for the classification of neutral atoms [as well as subgroups of $\mathrm{SO}(4,2)$ for the classification of ions] was also developed by A. O. Barut in 1972. The Barut approach was mainly based on the consideration of the atom as a system of electronic shells, while in the approach by Rumer, Fet and Konopel'chenko, further developed in collaboration with V. M. Byakov, Y. I. Kulakov and N. Sorokin, the atom is considered as an element of an irreducible representation of the group $\mathrm{SU}(2) \times$ $\mathrm{SO}(4,2)$.

Chapters 5 and 6 of the book constitute a review (in English) of the pioneering work achieved in the USSR (and mainly published in Russian) by Rumer, Fet, Konopel'chenko, Byakov, Kulakov and Sorokin between 1970 and 1979 on the periodic table. In this respect, the book is of great interest, from the theoretical viewpoint as well as the historical viewpoint, to the English-speaking community of physicists and chemists interested in the structure of matter. The references in the book concern mainly articles and books published before 1984 (with two exceptions corresponding to books published or reprinted in 1989 and 2000, probably discovered when the English version was composed). Therefore, the part on the classification of elementary particles (Chapter 5) is obsolete, although sufficient for understanding the transfer to atoms of methods used for strongly interacting particles. The part devoted to group theory and quantum mechanics is also sufficient for researchers and students accustomed to the use of Lie groups and Lie algebras, but for people not familiar with these notions further reading is necessary.

A great deal of work on the periodic table has been conducted since 1984. To fill the gap between 1984 and the present day the reader is advised to consult the books and articles listed below as further reading as well as references therein. In particular, in references (3)-(6), (8) and (9) the symmetry $\mathrm{SO}(4,2)$ is further investigated for atoms and molecules, respectively. References (10) and (11) contain many facets of the story of the periodic table of elements. The combined reading of the book by Fet and of references (1)(11) should give the reader a good understanding of the classification and analysis of the properties of atoms and molecules.

Further reading

(1) Rouvray, D. H. \& King, R. B. (2004). The Periodic Table: Into the 21st Century. Baldock: Research Studies Press.

(2) Rouvray, D. H. \& King, R. B. (2006). The Mathematics of the Periodic Table. New York: Nova Science.

(3) Kibler, M. R. (2004). Classifying chemical elements and particles: from the atomic to the subatomic world, see reference (1), pp. 297-329; arXiv:quant-ph/0310155.

(4) Kibler, M. R. (2006). A group-theoretical approach to the periodic table: old and new developments, see reference (2), pp. 237-263; arXiv:quant-ph/0503039.

(5) Kibler, M. R. (2004). On the use of the group $S O(4,2)$ in atomic and molecular physics. Molec. Phys. 102, 1221-1229; arXiv:quant-ph/0409209.

(6) Kibler, M. R. (2004). On a group-theoretical approach to the periodic table of chemical elements. In Symmetry Methods in Physics XI, edited by Č. Burdík, O. Navrátil \& S. Pošta. Dubna: Joint Institute for Nuclear Research; arXiv:quant-ph/ 0408104.

(7) Belokolos, E. D. (2017). Mendeleev table: a proof of Madelung rule and atomic Tietz potential. Symm. Integr. Geom. Methods Appl. (SIGMA), 13, 038.

(8) Zhuvikin, G. V. \& Hefferlin, R. (1994). Symmetry principles for periodic systems of molecules. Joint Report SC/ SPBU-1. Southern College, Collegedale, Tennessee, USA, and Saint Petersburg University, Russia.

(9) Carlson, C. M., Hefferlin, R. A. \& Zhuvikin, G. V. (1995). Analysis of group theoretical periodic systems of molecules using tabulated data. Joint Report SC/SPBU-2. Southern College, Collegedale, Tennessee, USA, and Saint Petersburg University, Russia.

(10) Scerri, E. (2007). The Periodic Table: Its Story and Its Significance. New York: Oxford University Press.

(11) Kibler, M. R. (2007). From the Mendeleev periodic table to particle physics and back to the periodic table. Found. Chem. 9, 221-234; arXiv: quant-ph/0611287. 